\title{
I, nanorobot
}

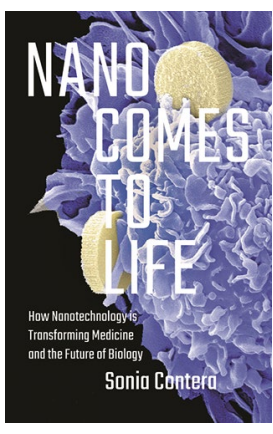

\section{Nano Comes to Life \\ By Sonia Contera \\ PRINCETON \\ UNIVERSITY PRESS: \\ 2019. 240 PP. $€ 22$}

ionanotechnology is the ultimate marriage of physics and biology, which makes this field a multidisciplinary challenge and often hard to define and communicate. In her new book, Nano Comes to Life, Sonia Contera, a professor of biological physics at the University of Oxford, zooms into this field to explore the key technological and scientific advances that have enabled biological research and manipulation at the nanoscale, highlighting how the multidisciplinarity inherent to nanotechnology led to major scientific breakthroughs in biology and medicine. Being a physicist herself, Contera argues that the arrival of nanotechnology has opened up the world of biology to physicists - and indeed, in nanotechnology, biological problems are tackled differently than in classic biology research: less biochemically, you could say. She calls this quantitative, more mathematical approach 'the physics of life'.

Contera starts her journey down to the nanoscale with the story of how X-rays enabled the identification of the structure of DNA. Undoubtedly, the collaboration leading to this discovery is a beautiful example of multidisciplinarity in science (although overshadowed by the fact that Rosalind Franklin's key contribution was not awarded with the Nobel prize), and marks a key milestone in biological research. However, it took many years - until the conclusion of the Human Genome Project - for us to understand that DNA is only the beginning and that the gene-reductionist view, which has long been a central dogma in biology, is far too simplistic to explain how a cell, and thus, our entire body, functions. And this is where physics, mathematics and engineering come in.
It is natural to discuss how the invention of the scanning tunnelling microscope marks the beginning of the field for many people, and how that was followed by the construction of the first atomic force microscope. Contera describes one of the most beautiful examples of how this technique was used to understand biology on a molecular level: the walk of myosins. Myosins are proteins that move along the skeleton of cells carrying cargo to different cellular compartments. If you have never seen myosin proteins walk in a cell, I highly recommend the $3 \mathrm{D}$ computer graphics animation The Inner Life of The Cell (https:// go.nature.com/37Xb7BB), which was made by scientists and illustrators at Harvard University. A major discovery made possible by nanotechnology.

In analogy to the movie Powers of Ten (https://go.nature.com/2UmnIdu) - which zooms in and out of the Universe by the power of ten - Contera then zooms out and focuses on emerging functions of biomolecules, as well as cellular signalling beyond biochemistry. She describes the work by Dennis Discher's lab, in which they describe how the mechanical properties of a substrate can direct mesenchymal stem cell fate - an important milestone in mechanobiology, in which the atomic force microscope plays a central role as a tool to investigate the mechanics of cells and their microenvironment. Back then, this was a major discovery, as it challenged the biochemistry-heavy view of biology. Now, we know that mechanics (and many other physical properties) play a role at every scale, and substantially contribute to cell and molecule fate, although the degree to which this happens remains an ongoing debate.

Moving from nanotechnological tools to nanotech design, the next part of the book is dedicated to protein and DNA nanotechnology, exploring proteins and nucleic acids as building blocks for the design of nanostructures, such as DNA origami and nanorobots. This is certainly one of the most active and challenging areas in nanotechnology, with an immense potential for drug delivery and for the development of alternatives to antibodies. DNA nanotechnology greatly benefited from computational methods that allow the rational design of specific patterns and structures. Similarly, in protein nanotechnology, which uses proteins and peptides to build nanostructures, the key breakthrough was achieved by simulating and predicting protein folding and assembly - a method pioneered by David Baker's lab at the University of Washington - that is now used in drug discovery and design, and even for the design of artificial proteins that can be used as nanodevices.

One of the great promises of nanotechnology was to revolutionize drug delivery. Contera takes a critical look at the history and achievements of nanomedicine for the targeted delivery of therapeutics. In this sense, the book comes at an interesting time, as the research community is currently passionately debating how to improve the clinical translatability of nanocarriers and devices, as Nature Nanotechnology highlighted recently (Nat. Nanotechnol. 14, 1083; 2019). Despite several clinical setbacks, Contera remains optimistic, and specifically mentions cancer immunotherapy and gene editing as key fields that will benefit from nanotechnology. However, she also emphasizes that more knowledge of the biology and physics of the delivery process will be needed to deliver on the promise of nanomedicine.

Contera ends her journey through the world of nanotechnology with an epilogue entitled 'Biology becomes physics'. While this is indeed a brave heading (and I am writing this as a bioengineering-trained Nature Nanotechnology editor sitting right next to the Nature Physics team), she means the importance of collaboration and collective narratives; that is, the responsibility of scientists to work together beyond disciplines, communicate their science and embrace equality to be able to responsibly use and apply the current and future nanotechnological advances. Nanotechnology has certainly challenged the independence of established scientific communities, and requires, by its very nature, the collaboration of different, sometimes quite distant, disciplines. But that is what makes it so exciting!

\footnotetext{
Reviewed by Christine-Maria Horejs

Senior Editor, Nature Nanotechnology.

e-mail: christine.horejs@nature.com
}

Published online: 6 March 2020

https://doi.org/10.1038/s41567-020-0820-1 